\title{
A study of dengue fever in pregnancy and its maternal and fetal prognosis
}

\author{
Hanslata Gehlot ${ }^{1 *}$, Om Prakash Yadav ${ }^{1}$, Seema Sharma ${ }^{1}$, Girdhar Gopal Nagar ${ }^{1}$, \\ Ayna Yadav ${ }^{2}$, Prabhu Prakash Gupta ${ }^{3}$
}

${ }^{1}$ Department of Obstetrics and Gynecology, ${ }^{2}$ Department of Anaesthesiology, ${ }^{3}$ Department of Microbiology, S. N. Medical College, Jodhpur, Rajasthan, India

Received: 24 April 2017

Revised: 24 June 2017

Accepted: 27 June 2017

\section{*Correspondence: \\ Dr. Hanslata Gehlot, \\ E-mail: pummyia@gmail.com}

Copyright: (c) the author(s), publisher and licensee Medip Academy. This is an open-access article distributed under the terms of the Creative Commons Attribution Non-Commercial License, which permits unrestricted non-commercial use, distribution, and reproduction in any medium, provided the original work is properly cited.

\section{ABSTRACT}

Background: Dengue is a vector borne viral disease. Female Anopheles mosquito is the vector for the disease. Recently, there is an increase in the incidence of dengue fever in adult population in South Asian countries. With increasing rate of adult dengue fever victims, the number of infected pregnant women has also been increased. Dengue, during pregnancy may be associated with various complications, including abortions, preterm delivery, maternal mortality, low birth weight, neonatal admissions and fetal anomalies. Timely intervention can improve the maternal as well as fetal outcome. This study was aimed to assess the clinical profile, maternal and fetal outcome of dengue fever during pregnancy.

Methods: The study was carried out on 25 pregnant females diagnosed and serologically confirmed to have dengue fever and were admitted in Umaid hospital, associated to Dr. S. N. Medical College Jodhpur, Rajasthan, India. Patients were included irrespective of the period of gestation of contracting the disease. Serological testing for dengue virus specific antigen and antibody was done for the diagnosis of dengue fever. The World Health Organization (WHO) classification and case definitions 2009 were used to categorize the dengue patients. A predesigned proforma was used to collect data related to maternal and fetal consequences both during pregnancy and at birth, as well as the effect on the newborn. Informed and written consent was taken from all those who participated in the study.

Results: Thrombocytopenia (platelet count $<1.5 \mathrm{lakh} / \mathrm{mm}^{3}$ ) was found in $22(88 \%)$ patients out of which $6(24 \%)$ of them had platelet count below 20,000 cells $/ \mathrm{mm}^{3}$ and $3(12 \%)$ patients required platelet transfusion. Other complications observed were spontaneous abortions (4\%); preterm birth (16\%), oligohydramnios (8\%) and antepartum hemorrhage (4\%). One patient was admitted to Intensive Care Unit. Fetal distress and meconium stained amniotic fluid was observed in $16 \%$ and $12 \%$ patients respectively. Adverse fetal outcome was observed in form of low birth weight, prematurity. $8 \%$ of the babies required NICU admission and $4 \%$ were Intra Uterine Fetal Death (IUFD).

Conclusions: Maternal infection with the dengue virus during antenatal period represents a real risk of premature birth. Early onset or late onset in pregnancy appeared to have a bad prognosis. A high index of clinical suspicion is essential in any pregnant woman with fever during the epidemic. The treatment of dengue in pregnancy is mainly conservative as in non-pregnant adults. In case of high risk cases early referral to well-equipped health centres where technical, transfusion and intensive care facilities are available may prove lifesaving.

Keywords: Dengue fever, Fetal outcome, Maternal outcome, Thrombocytopenia 


\section{INTRODUCTION}

Dengue is one of the most rapidly spreading vector borne viral disease in the world. There is an increasing incidence of dengue in adult population in South Asian, South- East Asian and Latin American countries in recent years. The dengue virus comprises four distinct serotypes (DEN 1-4) but DEN-3 is frequently associated with severe disease. ${ }^{1}$ Majority of dengue patients have a mild self-limiting illness and a few progresses to a severe disease. ${ }^{2}$ As the incidence of dengue in adults increases, the incidence in pregnant women also increases. ${ }^{2}$

Intravenous rehydration is the treatment of choice, which can reduce the mortality in dengue to less than $1 \%$ of severe cases. ${ }^{1}$ Dengue poses a significant threat to pregnant women. In present study, we have studied the pattern of dengue in pregnancy and the impact of dengue on the natural course of pregnancy. The maternal and fetal outcomes were also analyzed.

The clinical severity of disease has a wide spectrum, and according to the World Health Organization (WHO) dengue classification scheme, there are four grades of dengue, ranging from uncomplicated dengue fever (DF) to dengue hemorrhagic fever (DHF) and devastating dengue shock syndrome (DSS).

DHF is currently defined by the following four WHO criteria:

- Fever or recent history of fever lasting 2-7 days.

- Any hemorrhagic manifestation.

- Thrombocytopenia (platelet count of $<100,000 / \mathrm{mm}^{3}$ ).

- Evidence of increased vascular permeability.

With the recent resurgences of the disease, an increasing the number of people including the pregnant women are affected. Dengue infection in pregnancy carries the risk of hemorrhage for both the mother and the new-born. Literature search reveals an increased incidence of preterm deliveries, low birth weight babies, preeclampsia and caesarean sections. Vertical transmission has also been noted.

The study was aimed to assess the clinical profile, maternal and fetal outcome of dengue fever during pregnancy.

\section{METHODS}

The study was carried out in Umaid hospital associated to Dr. S. N. Medical College, a tertiary care maternal and child health care hospital in Jodhpur, Rajasthan. All the pregnant women who presented with fever and serologically confirmed as dengue fever were included in the study, irrespective of their gestational age. The age, weight, gestational age, parity, detailed history, clinical features, complete blood count, liver function test, renal function test, coagulation profiles were recorded on admission and were repeated serially as and when required till discharge.

Dengue was diagnosed using ELISA method by detection of dengue virus specific NS 1 antigen and $\operatorname{IgM}, \operatorname{IgG}$ antibody. ${ }^{3,4}$ Primary dengue infection was diagnosed when only IgM dengue virus specific antibody was detectable and secondary dengue infection was diagnosed when pregnant women had detectable $\operatorname{IgM}$ and $\operatorname{IgG}$ dengue antibodies. ${ }^{3,4}$

The impact of dengue on the natural course of pregnancy and labour and the maternal and fetal outcomes were noted.

\section{RESULTS}

In present study, a total of 25 patients who were diagnosed serologically with dengue fever were included. The age of the pregnant women ranged from 19-36 years. All the patients had fever with a temperature ranging between $99^{\circ} \mathrm{F}-104.1^{\circ} \mathrm{F}$ during the first five days of the illness. Generalized myalgia was present in 21 (84\%) patients followed by headache and skin rash.

Table 1: Symptomatology of patients.

\begin{tabular}{|lll|}
\hline Symptoms & No. of patients & $\%$ of patients \\
\hline Fever & 25 & 100 \\
\hline Myalgia & 21 & 84 \\
\hline Headache & 17 & 68 \\
\hline Skin rash & 6 & 24 \\
\hline Petechia & 3 & 12 \\
\hline Gum bleeding & 1 & 4 \\
\hline Antepartum & 1 & 4 \\
hemorrhage & 1 & 4 \\
\hline Abdominal pain & 1 & 4 \\
\hline Persistent vomiting & 1 & \\
\hline
\end{tabular}

Table 2: Serological reporting of dengue cases.

\begin{tabular}{|ll|}
\hline Biological test & No. of cases \\
\hline Dengue NS1 positive & 18 \\
\hline Dengue Ig M positive & 04 \\
\hline Only Ig G positive & 3 \\
\hline
\end{tabular}

According to Table 2, 18 patients were NS1 positive, 04 were Ig M positive and rest of the three were only Ig G positive. Two patients were found to have concurrent Widal positivity.

Table 3: Gestational age at diagnosis of dengue.

\begin{tabular}{|lll|}
\hline Gestational age (weeks) & No. of patients & $\%$ \\
\hline$<13$ & 1 & 4 \\
\hline $13-28$ & 4 & 16 \\
\hline$>28$ & 20 & 80 \\
\hline Total & $\mathrm{N}=25$ & 100 \\
\hline
\end{tabular}


Table 3 shows the distribution of gestational age at which diagnosis of dengue was made. $80 \%$ of the cases were affected in their third trimester, while only $20 \%$ of them were affected in first or second trimester.

Table 4: Severity of thrombocytopenia.

\begin{tabular}{|lll|}
\hline Platelet counts/cubic mm & No. of patients & $\%$ \\
\hline$<20,000$ & 6 & 24 \\
\hline $20,000-50,000$ & 9 & 36 \\
\hline $50,000-1$ lac & 3 & 12 \\
\hline 1 lac-1.5 lac & 4 & 16 \\
\hline$>1.5$ lac & 3 & 12 \\
\hline Total & $\mathrm{N}=25$ & 100 \\
\hline
\end{tabular}

Thrombocytopenia was present in almost $100 \%$ of the patients. In $60 \%$ of the patients, platelets were less than $50,000 / \mathrm{mm}^{3}$, while in 6 cases $(24 \%)$ platelets were less than $20,000 / \mathrm{mm}^{3}$.

Table 5: Outcome of pregnancy.

\begin{tabular}{|l|l|l|}
\hline Delivery & No. of patients & $\%$ \\
\hline Term vaginal delivery & 9 & 36 \\
\hline Preterm vaginal delivery & 8 & 32 \\
\hline Pregnancy continues & 4 & 16 \\
\hline Cesarean section & 2 & 8 \\
\hline Spontaneous abortion & 1 & 4 \\
\hline IUD vaginal delivery & 1 & 4 \\
\hline Total & $\mathrm{n}=25$ & 100 \\
\hline
\end{tabular}

Table 5 reveals the outcome of pregnancy in dengue cases. 9 patients delivered at term, while 8 of them had preterm delivery. 2 of them underwent caesarean section, 1 had an intrauterine fetal death, while spontaneous abortion was seen in 1 patient. In rest of the 4 patients, pregnancy continued.

\section{Table 6: Neonatal outcome.}

\begin{tabular}{|ll|}
\hline Neonatal outcomes & \\
\hline Prematurity & $7(28 \%)$ \\
\hline Low birth weight & $4(16 \%)$ \\
\hline Pregnancy continued & $4(16 \%)$ \\
\hline NICU admission & $2(8 \%)$ \\
\hline $\begin{array}{l}\text { Neonatal thrombocytopenia and related } \\
\text { complications }\end{array}$ & $1(4 \%)$ \\
\hline Normal & $7(28)$ \\
\hline
\end{tabular}

In terms of neonatal outcome, $28 \%$ were premature, $16 \%$ had low birth weight, $8 \%$ of them required neonatal admission, and 1 newborn presented with neonatal thrombocytopenia and related complications.

\section{DISCUSSION}

Dengue fever in pregnancy creates anxiety amongst the treating obstetrician and also amongst the patients and their relatives for the fear of bleeding tendencies. The outbreak of dengue is common during the monsoon and post monsoon season. ${ }^{5,6}$ Dengue in pregnancy requires early diagnosis and treatment and it should be considered differential diagnosis of fever during epidemics. ${ }^{7}$

A high index of clinical suscpicion is essential in any pregnant female with fever during epidemics especially in endemic areas like Kerla. ${ }^{8}$

The age of patients ranged from 19-36 years and 4 (16\%) patients presented with dengue in the second trimester. Number of pregnant women who presented with primary dengue infection were higher ( $72 \%$ cases) than the number of secondary dengue infection 7 cases $(28 \%$ cases). Most common presenting symptom other than fever was myalgia followed by headache and skin rash.

In our study 25 pregnant women were included. The age of the pregnant women ranged from 19-36 years. All the patients had fever with a temperature between $99^{\circ} \mathrm{F}-104.1$ during the first 1-5 days of the illness.

Fever with Generalized myalgia was present in 21 (84\%) patients (Table 1). Four patients were diagnosed with dengue in the second trimester (Table 3). Number of pregnant women who had dengue \pm warning signs and severe dengue were $23(92 \%)$ and $02(8 \%)$ respectively. Severe thrombocytopenia (platelet count <20000/cubic $\mathrm{mm}$ ) was seen in $24 \%$ cases (Table 4). Renal function test was normal in all the patients. Severe dengue was seen in two patients $(12.5 \%)$ in the third trimester, who required Single Donor Platelet (SDP) transfusion.

Out of these 25 diagnosed patients only 4 required platelet transfusions, rest were managed conservatively. One had profuse vaginal bleeding with passage of blood clots per vaginum. She delivered an intrauterine dead fetus and was managed in ICU. She was transfused with 2 units of packed cells, 1 unit SDP and 6 units of RDP. There was mild elevation of liver transaminases in 08 (32\%) patients. Preterm delivery was seen in $08(32 \%)$ patients (Table 5). The weight of the baby at birth was less than $2.5 \mathrm{~kg}$ in $04(16 \%)$ women (Table 6). Symptomatic dengue infection during pregnancy may increase the risk of PTB and LBW for infants. ${ }^{9}$

\section{CONCLUSION}

This study highlights the clinical pattern and the natural course of dengue in pregnancy. The clinical presentation of dengue in pregnancy is almost similar to dengue in non-pregnant adults. There is increased association of dengue with preterm delivery and low birth weight. Dengue Hemorrhagic fever may present with features similar to Preeclampsia and HELLP Syndrome. Serological tests aid in confirming the diagnosis of DHF. The gestational age at presentation of dengue fever appeared to be significant. Early onset or late onset in pregnancy appeared to have a bad prognosis. Conservative treatment should be given unless there are 
complications so Conservative medical and obstetrical management is the treatment of choice. ${ }^{10}$

Funding: No funding sources

Conflict of interest: None declared

Ethical approval: The study was approved by the Institutional Ethics Committee

\section{REFERENCES}

1. WHO. Dengue: guidelines for diagnosis, treatment, prevention and control. A joint publication of the World Health Organization (WHO) and the Special Programme for Research and Training in Tropical Diseases (TDR). New edition 2009

2. Chitra TV, Panicker S. Maternal and fetal outcome of dengue fever in pregnancy. $\mathrm{J}$ Vector Borne Dis. 2011;48(4):210.

3. N Bhattacharya, $\mathrm{H}$ Mukherjee, $\mathrm{R}$ Naskar, $\mathrm{S}$ Talukdar. Serological diagnosis of dengue in laboratory practice in Kolkata. Indian $\mathbf{J}$ Med Microbiol. 2014;32(3):277-80.

4. Vazquez S, Perez AB, Ruiz D, Rodriguez R, Pupo M, Calzada N, González L, González D, Castro O, Serrano T, Guzmán MG. Serological markers during dengue 3 primary and secondary infections. J Clin Virol. 2005;33(2):132-7.

5. Gupta E, Dar L, Narang P, Srivastava VK. Serodiagnosis of dengue during an outbreak at a tertiary care hospital in Delhi. Indian J Med Res. 2005;121:36-8.

6. Gunasekaran P, Kaveri K, Mohana S, Arunagiri K, Babu BS, Priya PP et al Dengue disease status in Chennai (2006-2008): A retrospective analysis. Indian J Med Res. 2011;133(3):322-5.

7. Kariyawasam S, Senanayake H. Degue infections during pregnancy: case series from a tertiary care hospital in Sri lanka. J Infect Dev Ctries. 2010;4(11):767-75.

8. Kanakalatha DH, Radha S, Nambisan B. Maternal and fetal outcome of dengue fever during pregnancy. Int J Reprod Contracept Obstet Gynecol. 2016;5(11):3959-64.

9. Friedman EE, Dallah F, Harville EW, Myers L, Buekens P, Breart $G$ et al. Symptomatic dengue infection during pregnancy and infant outcomes: a retrospective cohort study. PLoS Neglected Trop Dis. 2014;8(10):e3226.

10. Phupong V. Dengue fever in pregnancy: a case report. BMC Pregnancy Childbirth. 2001;1(1):7.

Cite this article as: Gehlot $\mathrm{H}$, Yadav OP, Sharma S, Nagar GG, Yadav A, Gupta PP. A study of dengue fever in pregnancy and its maternal and fetal prognosis. Int J Reprod Contracept Obstet Gynecol 2017;6:3414-7. 